\title{
DISTRIBUTION-VALUED ITERATED GRADIENT AND \\ CHAOTIC DECOMPOSITIONS OF POISSON JUMP \\ TIMES FUNCTIONALS
}

\author{
NicOlas PRIVAULT
}

Abstract

We define a class of distributions on Poisson space which allows to iterate a modification of the gradient of $[\mathbf{1}]$. As an application we obtain, with relatively short calculations, a formula for the chaos expansion of functionals of jump times of the Poisson process.

\section{Introduction}

Let $\left(N_{t}\right)_{t \in \mathbb{R}_{+}}$be a standard Poisson process with jump times $\left(T_{k}\right)_{k \geq 1}$, and $T_{0}=0$. The underlying probability space is denoted by $(\Omega, \mathcal{F}, P)$, so that $L^{2}(\Omega, \mathcal{F}, P)$ is the space of square-integrable functionals of $\left(N_{t}\right)_{t \in \mathbb{R}_{+}}$. Any $F \in L^{2}(\Omega, \mathcal{F}, P)$ can be expanded into the series

$$
F=E[F]+\sum_{n=1}^{\infty} \frac{1}{n !} I_{n}\left(f_{n}\right)
$$

where $I_{n}\left(f_{n}\right)$ is the iterated stochastic integral

$$
I_{n}\left(f_{n}\right)=n ! \int_{0}^{\infty} \int_{0}^{t_{n}} \cdots \int_{0}^{t_{2}} f_{n}\left(t_{1}, \ldots, t_{n}\right) d\left(N_{t_{1}}-t_{1}\right) \cdots d\left(N_{t_{n}}-t_{n}\right)
$$

of the symmetric function $f_{n} \in L^{2}\left(\mathbb{R}_{+}^{\circ n}\right)$ (stochastic integrals are taken in the Itô sense, thus diagonal terms have no influence in the above expression), with the isometry

$$
\begin{gathered}
\left\langle I_{n}\left(f_{n}\right), I_{m}\left(g_{m}\right)\right\rangle_{L^{2}(\Omega)}=n ! 1_{\{n=m\}}\left\langle f_{n}, g_{m}\right\rangle_{L^{2}\left(\mathbb{R}_{+}, d t\right)^{\circ n}}, \\
f_{n} \in L^{2}\left(\mathbb{R}_{+}, d t\right)^{\circ n}, \quad g_{m} \in L^{2}\left(\mathbb{R}_{+}, d t\right)^{\circ m} .
\end{gathered}
$$

2000 Mathematics Subject Classification. 60J75, 60H05.

Key words. Poisson process, stochastic analysis, chaotic decompositions. 
If $f_{n}$ is not symmetric we let $I_{n}\left(f_{n}\right)=I_{n}\left(\tilde{f}_{n}\right)$, where $\tilde{f}_{n}$ denotes the symmetrization of $f_{n}$ in $n$ variables, hence (1) can be written as

$$
F=E[F]+\sum_{n=1}^{\infty} I_{n}\left(f_{n} 1_{\Delta_{n}}\right)
$$

where

$$
\Delta_{n}=\left\{\left(t_{1}, \ldots, t_{n}\right) \in \mathbb{R}_{+}^{n}: 0 \leq t_{1}<\cdots<t_{n}\right\} .
$$

Let $D: L^{2}(\Omega) \longrightarrow L^{2}\left(\Omega \times \mathbb{R}_{+}\right)$denote the linear unbounded operator defined on multiple stochastic integrals as

$$
D_{t} I_{n}\left(f_{n}\right)=n I_{n-1}\left(f_{n}(*, t)\right), \quad \text { a.e. } t \in \mathbb{R}_{+} .
$$

The formula of Y. Ito [3, Relations (7.4) and (7.5), pp. 26-27], allows in principle to compute $f_{n}$ as

$$
f_{n}\left(t_{1}, \ldots, t_{n}\right)=E\left[D_{t_{1}} \cdots D_{t_{n}} F\right], \quad \text { a.e. } t_{1}, \ldots, t_{n} \in \mathbb{R}_{+} .
$$

Given the probabilistic interpretation of $D$ as a finite difference operator (cf. [3] and [6]), we have for $F=f\left(T_{1}, \ldots, T_{d}\right)$ :

$$
\begin{gathered}
D_{t} F=\sum_{k=1}^{k=d} 1_{] T_{k-1}, T_{k}\right]}(t)\left(f\left(T_{1}, \ldots, T_{k-1}, t, T_{k}, \ldots, T_{d-1}\right)-f\left(T_{1}, \ldots, T_{d}\right)\right), \\
t \in \mathbb{R}_{+},
\end{gathered}
$$

thus $D_{t_{1}} \cdots D_{t_{n}} F$ is well defined and explicit computations can be carried out but may be complicated due to the recursive application of a finite difference operator, cf. [4]. See [8] for an elementary approach using only orthogonal expansions in Charlier polynomials.

On the other hand, the gradient $\tilde{D}$ of $[\mathbf{1}]$ (see also [2]), defined as

$$
\tilde{D}_{t}=-\sum_{k=1}^{k=d} 1_{\left[0, T_{k}\right]}(t) \partial_{k} f\left(T_{1}, \ldots, T_{d}\right),
$$

has some properties in common with $D$, namely its adapted projection coincides with that of $D$, and in particular we have

$$
E\left[D_{t} F\right]=E\left[\tilde{D}_{t} F\right], \quad t \in \mathbb{R}_{+} .
$$

Since the operator $\tilde{D}$ has the derivation property it is easier to manipulate than the finite difference operator $D$ in recursive computations. Its disadvantage is that it can not be iterated in $L^{2}$ due to the non-differentiability of $1_{\left[0, T_{k}\right]}(t)$ in $T_{k}$, thus an expression such as $E\left[\tilde{D}_{t_{1}} \cdots \tilde{D}_{t_{n}} F\right]$ makes a priori no sense, moreover $E\left[\tilde{D}_{t_{1}} \cdots \tilde{D}_{t_{n}} F\right]$ may differ from $E\left[D_{t_{1}} \cdots D_{t_{n}} F\right]$ for $n \geq 2$ (see Relations (13) and (14) below). 
In [7] the combined use of $D^{n}$ and $\tilde{D}$ in $L^{2}$ sense has led to the computation of the expansion of the jump time $T_{d}, d \geq 1$. A direct calculation using only the operator $D$ can be found in [5], concerning a Poisson process on a bounded interval.

In this paper we show that the gradient $\tilde{D}$ can be iterated in a precise sense of distributions on Poisson space, to be introduced in Section 3. For example we have for $\left(t_{1}, \ldots, t_{n}\right) \in \Delta_{n}$ :

$$
\begin{aligned}
\tilde{D}_{t_{1}} \cdots \tilde{D}_{t_{n}} f\left(T_{d}\right) & =(-1)^{n} f^{(n)}\left(T_{d}\right) 1_{\left[0, T_{d}\right]}\left(t_{1} \vee \cdots \vee t_{n}\right) \\
& +(-1)^{n} 1_{\left\{0 \leq t_{1}, \ldots, t_{n-1}<t_{n}\right\}} \sum_{j=1}^{n-1} f^{(n-j)}\left(t_{n}\right) \delta_{t_{n}}^{(j-1)}\left(T_{d}\right),
\end{aligned}
$$

where $\delta_{t_{n}}\left(T_{d}\right)$ is a generalized functional, i.e. the composition of the Dirac distribution $\delta_{t_{n}}$ at $t_{n}$ with the jump time $T_{d}$, cf. Proposition $3, f^{(n)}$ denotes the $n$-th derivative of the function $f \in \mathcal{C}_{b}^{\infty}\left(\mathbb{R}_{+}\right)$, and $t_{1} \vee \cdots \vee t_{n}=$ $\max \left(t_{1}, \ldots, t_{n}\right)$. Moreover we obtain the equality

$$
\begin{aligned}
E\left[D_{t_{1}} \cdots D_{t_{n}} F \mid \mathcal{F}_{a}\right]=E\left[\tilde{D}_{t_{1}} \cdots \tilde{D}_{t_{n}} F \mid \mathcal{F}_{a}\right], & \\
& 0 \leq a<t_{1}<\cdots<t_{n}, \quad n \geq 2,
\end{aligned}
$$

where we make sense of the conditional expectation $E\left[\tilde{D}_{t_{1}} \cdots \tilde{D}_{t_{n}} F \mid \mathcal{F}_{a}\right]$ using the pairing $\langle\cdot, \cdot\rangle$ between distributions and test functions. This implies

$$
f_{n}\left(t_{1}, \ldots, t_{n}\right)=E\left[\tilde{D}_{t_{1}} \cdots \tilde{D}_{t_{n}} F\right], \quad 0<t_{1}<\cdots<t_{n} .
$$

This gives an expression for the decomposition of $f\left(T_{1}, \ldots, T_{n}\right), f \in$ $\mathcal{C}_{b}^{\infty}\left(\Delta_{d}\right)$, with relatively short computations, cf. Proposition 6 , for example

$$
f\left(T_{d}\right)=\sum_{n=0}^{\infty} I_{n}\left(h_{n} 1_{\Delta_{n}}\right)
$$

with

$$
\begin{aligned}
& h_{n}\left(t_{1}, \ldots, t_{n}\right)=E\left[\tilde{D}_{t_{1}} \cdots \tilde{D}_{t_{n}} f\left(T_{d}\right)\right] \\
&=(-1)^{n} \int_{t_{n}}^{\infty} f^{(n)}(t) p_{d-1}(t) d t+(-1)^{n} \sum_{j=1}^{j=n-1}(-1)^{j-1} f^{(n-j)}\left(t_{n}\right) p_{d-1}^{(j-1)}\left(t_{n}\right), \\
& 0<t_{1}<\cdots<t_{n}, \text { where } p_{d-1}(t)=\frac{t^{d-1}}{(d-1) !} e^{-t}, t \in \mathbb{R}_{+}, d \geq 1 .
\end{aligned}
$$




\section{Integration by parts}

In this section we review the definition of the three main gradient operators on Poisson space, and present an elementary derivation of integration by parts formulas. All $\mathcal{C}^{\infty}$ functions on $\Delta_{d}$ are extended by continuity to the closure of $\Delta_{d}$.

Definition 1. Let $a \geq 0$. Let $\mathcal{S}_{d}\left(\Omega \times\left[a, \infty\left[^{l}\right)\right.\right.$ denote the test function space

$$
\begin{aligned}
\mathcal{S}_{d}\left(\Omega \times[a, \infty]^{l}\right)=\left\{h_{1} \otimes \cdots \otimes\right. & h_{l} \otimes f\left(T_{1}, \ldots, T_{d}\right): \\
& \quad f \in \mathcal{C}_{b}^{\infty}\left(\Delta_{d}\right), h_{1}, \ldots, h_{l} \in \mathcal{C}_{b}([a, \infty[)\},
\end{aligned}
$$

with $\mathcal{S}_{d}(\Omega)=\mathcal{S}_{d}\left(\Omega \times \mathbb{R}_{+}^{0}\right)$ for $l=0$.

We recall that if $f \in L^{2}\left(\Delta_{d}, e^{-t_{d}} d t_{1} \cdots d t_{d}\right)$ then

$$
E\left[f\left(T_{1}, \ldots, T_{d}\right)\right]=\int_{0}^{\infty} e^{-t_{d}} \int_{0}^{t_{d}} \cdots \int_{0}^{t_{2}} f\left(t_{1}, \ldots, t_{d}\right) d t_{1} \cdots d t_{d}
$$

which follows e.g. from the fact that $\left(\tau_{n}\right)_{n \geq 1}=\left(T_{n}-T_{n-1}\right)_{n \geq 1}$ is a family of independent exponential random variables.

\subsection{Intrinsic gradient.}

The intrinsic gradient $\hat{D}$ on Poisson space is defined on $\mathcal{S}_{d}(\Omega)$ as

$$
\hat{D}_{t} F=\sum_{k=1}^{k=d} 1_{\left\{T_{k}\right\}}(t) \partial_{k} f\left(T_{1}, \ldots, T_{d}\right), \quad d N_{t} \text {-a.e. }
$$

with $F=f\left(T_{1}, \ldots, T_{d}\right), f \in \mathcal{C}_{b}^{\infty}\left(\Delta_{d}\right)$, where $\partial_{k} f$ represents the partial derivative of $f$ with respect to its $k$-th variable, $1 \leq k \leq d$.

Lemma 1. Let $F \in \mathcal{S}_{d}(\Omega)$ and $h \in \mathcal{C}_{b}^{1}\left(\mathbb{R}_{+}\right)$with $h(0)=0$. We have the integration by parts formula

(2) $E\left[\langle\hat{D} F, h\rangle_{L^{2}\left(\mathbb{R}_{+}, d N_{t}\right)}\right]=E\left[F U_{h^{\prime}}^{d}\right]=-E\left[F \int_{0}^{\infty} h^{\prime}(t) d\left(N_{t}-t\right)\right]$,

where $U_{h^{\prime}}^{d}=-\left(\sum_{k=1}^{k=d} h^{\prime}\left(T_{k}\right)-\int_{0}^{T_{d}} h^{\prime}(t) d t\right) \in \mathcal{S}_{d}(\Omega)$. 
Proof: We have by integration by parts on $\Delta_{d}$ :

$$
\begin{aligned}
& E\left[\langle\hat{D} F, h\rangle_{L^{2}\left(\mathbb{R}_{+}, d N_{t}\right)}\right]=\sum_{k=1}^{k=d} \int_{0}^{\infty} \int_{0}^{t_{d}} \cdots \int_{0}^{t_{2}} e^{-t_{d}} h\left(t_{k}\right) \partial_{k} f\left(t_{1}, \ldots, t_{d}\right) d t_{1} \cdots d t_{d} \\
& =\int_{0}^{\infty} e^{-t_{d}} \int_{0}^{t_{d}} \cdots \int_{0}^{t_{2}} h\left(t_{1}\right) \partial_{1} f\left(t_{1}, \ldots, t_{d}\right) d t_{1} \cdots d t_{d} \\
& +\sum_{k=2}^{k=d} \int_{0}^{\infty} e^{-t_{d}} \int_{0}^{t_{d}} \cdots \int_{0}^{t_{k+1}} h\left(t_{k}\right) \frac{\partial}{\partial t_{k}} \int_{0}^{t_{k}} \int_{0}^{t_{2}} f\left(t_{1}, \ldots, t_{d}\right) d t_{1} \cdots d t_{d} \\
& -\sum_{k=2}^{k=d} \int_{0}^{\infty} e^{-t_{d}} \int_{0}^{t_{d}} \cdots \int_{0}^{t_{k+1}} h\left(t_{k}\right) \int_{0}^{t_{k}} \int_{0}^{t_{k-2}} \cdots \int_{0}^{t_{2}} f\left(t_{1}, \ldots, t_{k-2}, t_{k}, t_{k}, \ldots, t_{d}\right) \\
& d t_{1} \cdots d \hat{t}_{k-1} \cdots d t_{d} \\
& =-\int_{0}^{\infty} e^{-t_{d}} \int_{0}^{t_{d}} \cdots \int_{0}^{t_{2}} h^{\prime}\left(t_{1}\right) f\left(t_{1}, \ldots, t_{d}\right) d t_{1} \cdots d t_{d} \\
& +\int_{0}^{\infty} e^{-t_{d}} \int_{0}^{t_{d}} \cdots \int_{0}^{t_{3}} h\left(t_{2}\right) f\left(t_{2}, t_{2}, \ldots, t_{d}\right) d t_{2} \cdots d t_{d} \\
& -\sum_{k=2}^{k=d} \int_{0}^{\infty} e^{-t_{d}} \int_{0}^{t_{d}} \cdots \int_{0}^{t_{2}} h^{\prime}\left(t_{k}\right) f\left(t_{1}, \ldots, t_{d}\right) d t_{1} \cdots d t_{d} \\
& +\int_{0}^{\infty} e^{-t_{d}} h\left(t_{d}\right) \int_{0}^{t_{d}} \cdots \int_{0}^{t_{2}} f\left(t_{1}, \ldots, t_{d}\right) d t_{1} \cdots d t_{d} \\
& +\sum_{k=2}^{k=d-1} \int_{0}^{\infty} e^{-t_{d}} \int_{0}^{t_{d}} \cdots \int_{0}^{t_{k+1}} \int_{0}^{t_{k-1}} \cdots \int_{0}^{t_{2}} h\left(t_{k+1}\right) f\left(t_{1}, \ldots, t_{k-1}, t_{k+1}, t_{k+1}, \ldots, t_{d}\right) \\
& d t_{1} \cdots d \hat{t}_{k} \cdots d t_{d} \\
& -\sum_{k=2}^{k=d} \int_{0}^{\infty} e^{-t_{d}} \int_{0}^{t_{d}} \cdots \int_{0}^{t_{k}} \int_{0}^{t_{k-2}} \cdots \int_{0}^{t_{2}} h\left(t_{k}\right) f\left(t_{1}, \ldots, t_{k-2}, t_{k}, t_{k}, \ldots, t_{d}\right) d t_{1} \cdots d t_{d} \\
& =-\sum_{k=1}^{k=d} \int_{0}^{\infty} e^{-t_{d}} \int_{0}^{t_{d}} \cdots \int_{0}^{t_{2}} h^{\prime}\left(t_{k}\right) f\left(t_{1}, \ldots, t_{d}\right) d t_{1} \cdots d t_{d} \\
& +\int_{0}^{\infty} e^{-t_{d}} h\left(t_{d}\right) \int_{0}^{t_{d}} \cdots \int_{0}^{t_{2}} f\left(t_{1}, \ldots, t_{d}\right) d t_{1} \cdots d t_{d} \\
& =-E\left[F\left(\sum_{k=1}^{k=d} h^{\prime}\left(T_{k}\right)-\int_{0}^{T_{d}} h^{\prime}(t) d t\right)\right]
\end{aligned}
$$


where $d \hat{t}_{k}$ denotes the absence of $d t_{k}$. Concerning the second part of the equality it suffices to notice that if $k>d$,

$$
\begin{aligned}
E\left[F h^{\prime}\left(T_{k}\right)\right] & \\
= & \int_{0}^{\infty} e^{-t_{k}} h^{\prime}\left(t_{k}\right) \int_{0}^{t_{k}} \cdots \int_{0}^{t_{d}} \cdots \int_{0}^{t_{2}} f\left(t_{1}, \ldots, t_{d}\right) d t_{1} \cdots d t_{k} \\
= & \int_{0}^{\infty} e^{-t_{k}} h\left(t_{k}\right) \int_{0}^{t_{k}} \cdots \int_{0}^{t_{d}} \cdots \int_{0}^{t_{2}} f\left(t_{1}, \ldots, t_{d}\right) d t_{1} \cdots d t_{k} \\
& -\int_{0}^{\infty} e^{-t_{k-1}} h\left(t_{k-1}\right) \int_{0}^{t_{k-1}} \cdots \int_{0}^{t_{d}} \cdots \int_{0}^{t_{2}} f\left(t_{1}, \ldots, t_{d}\right) d t_{1} \cdots d t_{k-1} \\
= & E\left[F\left(h\left(T_{k}\right)-h\left(T_{k-1}\right)\right)\right]=E\left[F \int_{T_{k-1}}^{T_{k}} h^{\prime}(t) d t\right] .
\end{aligned}
$$

Relation (2) implies immediately for $F, G \in \mathcal{S}_{d}(\Omega)$ :

$$
\begin{aligned}
E\left[\langle\hat{D} F, h G\rangle_{L^{2}\left(\mathbb{R}_{+}, d N_{t}\right)}\right] & \\
& =E\left[\langle\hat{D}(F G), h\rangle_{L^{2}\left(\mathbb{R}_{+}, d N_{t}\right)}-F\langle\hat{D} G, h\rangle_{L^{2}\left(\mathbb{R}_{+}, d N_{t}\right)}\right] \\
& =E\left[F\left(G U_{h^{\prime}}^{d}-\langle h, \hat{D} G\rangle_{L^{2}\left(\mathbb{R}_{+}, d N_{t}\right)}\right)\right] \\
& =-E\left[F\left(G \int_{0}^{\infty} h^{\prime}(t) d\left(N_{t}-t\right)+\langle h, \hat{D} G\rangle_{L^{2}\left(\mathbb{R}_{+}, d N_{t}\right)}\right)\right] .
\end{aligned}
$$

\subsection{Damped gradient.}

Let $r(s, t)=-s \vee t$ denote the Green function associated to the Laplacian $\mathcal{L}$ on $\mathbb{R}_{+}$:

$$
\mathcal{L} f=-f^{\prime \prime}, \quad f \in \mathcal{C}_{c}^{\infty}(] 0, \infty[),
$$

i.e. we have, with $g=-f^{\prime \prime}$ :

$$
\int_{0}^{\infty} r(s, t) g(t) d t=-\int_{0}^{s} \int_{0}^{t} g(u) d u d t, \quad s \in \mathbb{R}_{+} .
$$

Definition 2. Given $F \in \mathcal{S}_{d}(\Omega), F=f\left(T_{1}, \ldots, T_{d}\right)$, we let

$$
r^{(1)}(s, t)=\frac{\partial}{\partial s} r(s, t)=-1_{]-\infty, s]}(t), \quad s, t \in \mathbb{R}_{+},
$$


and

$$
\tilde{D}_{t} F=\int_{0}^{\infty} r^{(1)}(s, t) \hat{D}_{s} F d N_{s}
$$

We have

$$
\tilde{D}_{t} F=\sum_{k=1}^{k=d} r^{(1)}\left(T_{k}, t\right) \partial_{k} f\left(T_{1}, \ldots, T_{d}\right)=-\sum_{k=1}^{k=d} 1_{\left[0, T_{k}\right]}(t) \partial_{k} f\left(T_{1}, \ldots, T_{d}\right) .
$$

In fact $\tilde{D}$ is (up to a minor modification) the gradient introduced in [1]. This presentation of $\tilde{D}$ using the Green function $r(s, t)$ is motivated by $[\mathbf{9}]$.

Proposition 1. We have for $F \in \mathcal{S}_{d}(\Omega)$ and $h \in \mathcal{C}_{c}\left(\mathbb{R}_{+}\right)$:

(3) $E\left[\langle\tilde{D} F, h\rangle_{L^{2}\left(\mathbb{R}_{+}, d t\right)}\right]=E\left[F U_{h}^{d}\right]=E\left[F \int_{0}^{\infty} h(t) d\left(N_{t}-t\right)\right]$, where $U_{h}^{d}=\sum_{k=1}^{k=d} h\left(T_{k}\right)-\int_{0}^{T_{d}} h(t) d t$.

Proof: We have

$$
\begin{aligned}
E\left[\langle\tilde{D} F, h\rangle_{L^{2}\left(\mathbb{R}_{+}, d t\right)}\right] & =E\left[\int_{0}^{\infty} \int_{0}^{\infty} r^{(1)}(s, t) \hat{D}_{s} F h(t) d N_{s} d t\right] \\
& =-E\left[\left\langle\hat{D} F, \int_{0}^{.} h(t) d t\right\rangle_{L^{2}\left(\mathbb{R}_{+}, d N_{t}\right)}\right] \\
& =E\left[F \int_{0}^{\infty} h(t) d\left(N_{t}-t\right)\right] .
\end{aligned}
$$

Relation (3) also implies that for $F, G \in \mathcal{S}_{d}(\Omega)$,

$$
\begin{aligned}
E\left[\langle\tilde{D} F, h G\rangle_{L^{2}\left(\mathbb{R}_{+}, d t\right)}\right] & \\
& =E\left[\langle\tilde{D}(F G), h\rangle_{L^{2}\left(\mathbb{R}_{+}, d t\right)}-F\langle\tilde{D} G, h\rangle_{L^{2}\left(\mathbb{R}_{+}, d t\right)}\right] \\
& =E\left[F\left(G U_{h}^{d}-\langle h, \tilde{D} G\rangle_{L^{2}\left(\mathbb{R}_{+}, d t\right)}\right)\right] \\
& =E\left[F\left(G \int_{0}^{\infty} h(t) d\left(N_{t}-t\right)-\langle h, \tilde{D} G\rangle_{L^{2}\left(\mathbb{R}_{+}, d t\right)}\right)\right] .
\end{aligned}
$$

\subsection{Finite difference gradient.}

For completeness we mention the gradient $D$ which is associated to the Fock space structure, and whose properties have been discussed in the introduction. 


\section{Distribution-valued gradient}

For $n \geq 2$ and $F \in \mathcal{S}_{d}(\Omega)$ we let $d N_{t_{1}} \otimes \cdots \otimes d N_{t_{n}}$-a.e.:

$$
\hat{D}_{t_{1}, \ldots, t_{n}}^{n} F=\sum_{1 \leq j_{1}, \ldots, j_{n} \leq d} 1_{\left\{T_{j_{1}}\right\}}\left(t_{1}\right) \cdots 1_{\left\{T_{j_{n}}\right\}}\left(t_{n}\right) \partial_{j_{1}} \cdots \partial_{j_{n}} f\left(T_{1}, \ldots, T_{d}\right) .
$$

This is not the $n$-th iteration of $\hat{D}$, in fact we have

$$
\left\|\hat{D}^{n} F\right\|_{L^{2}\left(\mathbb{R}_{+}, d N_{t}\right) \otimes n}^{2}=\sum_{1 \leq j_{1}, \ldots, j_{n} \leq d}\left(\partial_{j_{1}} \cdots \partial_{j_{n}} f\left(T_{1}, \ldots, T_{d}\right)\right)^{2} .
$$

Definition 3. Let $a \in \mathbb{R}_{+}$and $l \in \mathbb{N}$.

i) We denote by $\mathcal{S}_{d}^{\prime}\left(\Omega \times\left[a, \infty\left[^{l}\right)\right.\right.$ the space of continuous linear forms (distributions) on $\mathcal{S}_{d}\left(\Omega \times\left[a, \infty\left[^{l}\right)\right.\right.$, i.e. $F \in \mathcal{S}_{d}^{\prime}\left(\Omega \times\left[a, \infty\left[^{l}\right)\right.\right.$ if there exists $k \geq 0$ and $C>0$ such that

$\left|\left\langle F, h_{1} \otimes \cdots \otimes h_{l} \otimes G\right\rangle\right| \leq C \sum_{i=0}^{i=k}\left\|h_{1} \otimes \cdots \otimes h_{l}\right\|_{\infty}\left\|\hat{D}^{i} G\right\|_{L^{\infty}\left(\Omega, L^{2}\left(\mathbb{R}_{+}, d N_{t}\right)^{\otimes i}\right)}$, $G \in \mathcal{S}_{d}\left(\Omega \times \mathbb{R}_{+}^{l}\right), h_{1}, \ldots, h_{l} \in \mathcal{C}_{c}([a, \infty[)$.

ii) A sequence $\left(F_{n}\right)_{n \in \mathbb{N}} \subset \mathcal{S}_{d}^{\prime}\left(\Omega \times\left[a, \infty{ }^{l}\right)\right.$ is said to converge in $\mathcal{S}_{d}^{\prime}(\Omega \times$ $\left[a, \infty\left[^{l}\right)\right.$ if the sequence $\left(\left\langle F_{n}, G\right\rangle\right)_{n \in \mathbb{N}}$ converges to $\langle F, G\rangle$ for all $G \in \mathcal{S}_{d}\left(\Omega \times\left[a, \infty\left[{ }^{l}\right)\right.\right.$.

The notation $\langle\cdot, \cdot\rangle$ will be used to denote the pairing between $\mathcal{S}_{d}(\Omega \times$ $\left[a, \infty\left[{ }^{l}\right)\right.$ and $\mathcal{S}_{d}^{\prime}\left(\Omega \times\left[a, \infty\left[^{l}\right)\right.\right.$, for all values of $l \in \mathbb{N}$. Every $F \in \mathcal{S}_{d}(\Omega \times$ $\left.[a, \infty]^{l}\right)$ is identified to an element of $\mathcal{S}_{d}^{\prime}\left(\Omega \times\left[a, \infty\left[^{l}\right)\right.\right.$ by letting

$$
\langle F, G\rangle=\int_{\Omega}\langle F(\omega, \cdot), G(\omega, \cdot)\rangle_{L^{2}([a, \infty[, d t) \otimes l} P(d \omega), \quad G \in \mathcal{S}_{d}\left(\Omega \times\left[a, \infty\left[{ }^{l}\right) .\right.\right.
$$

The closability property in $L^{2}$ of the operator $\tilde{D}$ is a well-known statement which extends to distributions in $\mathcal{S}_{d}^{\prime}\left(\Omega \times\left[a, \infty\left[^{l}\right)\right.\right.$.

Proposition 2. Let $\left(F_{n}\right)_{n \in \mathbb{N}} \subset \mathcal{S}_{d}\left(\Omega \times\left[a, \infty\left[^{l}\right)\right.\right.$ such that

i) $\left(F_{n}\right)_{n \in \mathbb{N}}$ converges to 0 in $\mathcal{S}_{d}^{\prime}\left(\Omega \times\left[a, \infty\left[^{l}\right)\right.\right.$,

ii) $\left(\tilde{D} F_{n}\right)_{n \in \mathbb{N}}$ converges in $\mathcal{S}_{d}^{\prime}\left(\Omega \times\left[a, \infty\left[^{l+1}\right)\right.\right.$.

Then $\left(\tilde{D} F_{n}\right)_{n \in \mathbb{N}}$ converges to 0 in $\mathcal{S}_{d}^{\prime}\left(\Omega \times\left[a, \infty\left[^{l+1}\right)\right.\right.$.

Proof: For $l=0$ this is a direct consequence of the integration by parts formula (4), which shows that

$\left\langle\tilde{D} F_{n}, h G\right\rangle=E\left[F_{n}\left(G U_{h}^{d}-\langle\tilde{D} G, h\rangle_{L^{2}\left(\mathbb{R}_{+}, d t\right)}\right)\right], h \in \mathcal{C}_{c}\left(\left[a, \infty[), G \in \mathcal{S}_{d}(\Omega)\right.\right.$, with $G U_{h}^{d}-\langle\tilde{D} G, h\rangle_{L^{2}\left(\mathbb{R}_{+}, d t\right)} \in \mathcal{S}_{d}(\Omega)$. The generalization to $l \geq 1$ is straightforward. 
This proposition justifies the following extension of $\tilde{D}$ to generalized functionals.

Definition 4. Let $a \in \mathbb{R}_{+}$. We let $\mathbb{D}_{l}([a, \infty[)$ denote the subspace of $F \in \mathcal{S}_{d}^{\prime}\left(\Omega \times\left[a, \infty\left[^{l}\right)\right.\right.$ such that

i) there exists $\left(F_{n}\right)_{n \in \mathbb{N}} \subset \mathcal{S}_{d}\left(\Omega \times\left[a, \infty\left[^{l}\right)\right.\right.$ that converges to $F$ in $\mathcal{S}_{d}^{\prime}\left(\Omega \times\left[a, \infty\left[^{l}\right)\right.\right.$,

ii) $\left(\tilde{D} F_{n}\right)_{n \in \mathbb{N}}$ converges in $\mathcal{S}_{d}^{\prime}\left(\Omega \times\left[a, \infty\left[^{l+1}\right)\right.\right.$.

Given $F$ as above we define $\tilde{D} F$ as the limit in $\mathcal{S}_{d}^{\prime}\left(\Omega \times\left[a, \infty\left[{ }^{l+1}\right)\right.\right.$

$$
\tilde{D} F=\lim _{n \rightarrow \infty} \tilde{D} F_{n}, \quad F \in \mathbb{D}_{l}\left(\left[a, \infty[) \subset \mathcal{S}_{d}^{\prime}\left(\Omega \times\left[a, \infty\left[^{l}\right) .\right.\right.\right.\right.
$$

\section{Iterated gradient in distribution sense}

We let for $n \geq 2$ :

$$
r^{(n)}\left(T_{k}, t\right)=\partial_{1}^{n} r\left(T_{k}, t\right)=-\delta_{t}^{(n-2)}\left(T_{k}\right),
$$

in distribution sense, i.e. $r^{(n)}\left(T_{k}, t\right)$ belongs to $\mathcal{S}_{d}^{\prime}(\Omega)$ with for $k=$ $1, \ldots, d$, and $f \in \mathcal{C}_{b}^{\infty}\left(\Delta_{d}\right)$ :

$$
\begin{aligned}
& \left\langle r^{(n)}\left(T_{k}, t\right), f\left(T_{1}, \ldots, T_{d}\right)\right\rangle(-1)^{n+1} 1_{\{k<d\}} \\
& \times \int_{0}^{\infty} e^{-s_{d}} \int_{0}^{s_{d}} \cdots \int_{0}^{s_{k+2}}\left(\frac{\partial^{n-2}}{\partial s_{k}^{n-2}} \int_{0}^{s_{k}} \cdots \int_{0}^{s_{2}} f\left(s_{1}, \ldots, s_{d}\right) d s_{1} \cdots d s_{k-1}\right)_{\mid s_{k}=t} d s_{k+1} \cdots d s_{d} \\
& +(-1)^{n+1} 1_{\{k=d\}} \frac{\partial^{n-2}}{\partial t^{n-2}} \int_{0}^{t} \int_{0}^{s_{d-1}} \cdots \int_{0}^{s_{2}} e^{-t} f\left(s_{1}, \ldots, s_{d-1}, t\right) d s_{1} \cdots d s_{d-1}
\end{aligned}
$$

and for $n=1$ :

$$
\begin{aligned}
& \left\langle r^{(1)}\left(T_{k}, t\right), f\left(T_{1}, \ldots, T_{d}\right)\right\rangle \\
& =-\int_{0}^{\infty} \int_{0}^{s_{d}} \cdots \int_{0}^{s_{2}} e^{-s_{d}} 1_{[t, \infty[}\left(s_{k}\right) f\left(s_{1}, \ldots, s_{d}\right) d s_{1} \cdots d s_{d} .
\end{aligned}
$$

Let $\phi \in \mathcal{C}_{c}^{\infty}([-1,1]), \phi \geq 0$, such that $\int_{-1}^{1} \phi(t) d t=1$, and let

$$
\phi_{\varepsilon}(t)=\varepsilon^{-1} \phi\left(\varepsilon^{-1} t\right), \quad t \in \mathbb{R}, \quad \varepsilon>0 .
$$


Let $\phi_{\varepsilon} * r^{(n)}\left(T_{k}, t\right), n \geq 1$, denote the convolution of $\phi_{\varepsilon}$ with $r^{(n)}\left(T_{k}, t\right)$ in the first variable, i.e. for $n=1$ :

$$
\begin{aligned}
\phi_{\varepsilon} * r^{(1)}\left(T_{k}, t\right) & =-\int_{-\infty}^{\infty} \phi_{\varepsilon}(u) 1_{]-\infty, T_{k}-u\right]}(t) d u \\
& =-\int_{-\infty}^{\infty} \phi_{\varepsilon}\left(T_{k}+u\right) 1_{]-\infty, u]}(t) d u, \quad s, t \in \mathbb{R}_{+}
\end{aligned}
$$

and for $n \geq 2, l \in \mathbb{N}$ :

$$
\phi_{\varepsilon}^{(l)} * r^{(n)}\left(T_{k}, t\right)=\phi_{\varepsilon} * r^{(n+l)}\left(T_{k}, t\right)=-\phi_{\varepsilon}^{(n+l-2)}\left(T_{k}-t\right),
$$

which converges in $\mathcal{S}_{d}^{\prime}(\Omega)$ to $r^{(n+l)}\left(T_{k}, t\right)$ if $n+l \geq 1$ (i.e. to $-\delta_{t}^{(n+l-2)}\left(T_{k}\right)$ if $n+l \geq 2), k=1, \ldots, d$. Let $t_{1} \vee \cdots \vee t_{n}, t_{1}, \ldots, t_{n} \in \mathbb{R}_{+}$.

Proposition 3. Let $k \geq 1$ and $f \in \mathcal{C}_{b}^{\infty}\left(\mathbb{R}_{+}\right)$. Then for all $n \geq 1$, $\tilde{D}_{t_{1}} \cdots \tilde{D}_{t_{n}} f\left(T_{k}\right) \in \mathbb{D}_{0}\left(\mathbb{R}_{+}\right)$for a.a. $\left(t_{1}, \ldots, t_{n}\right) \in \mathbb{R}_{+}^{n}$, and

(5) $\quad \tilde{D}_{t_{1}} \cdots \tilde{D}_{t_{n}} f\left(T_{k}\right)=(-1)^{n} f^{(n)}\left(T_{k}\right) 1_{\left[0, T_{k}\right]}\left(t_{1} \vee \cdots \vee t_{n}\right)$

$+(-1)^{n} \sum_{j=1}^{n-1} 1_{\left\{t_{1} \vee \cdots \vee t_{j}<t_{j+1} \vee \cdots \vee t_{n}\right\}} f^{(n-j)}\left(t_{j+1} \vee \cdots \vee t_{n}\right) \delta_{t_{j+1} \vee \cdots \vee t_{n}}^{(j-1)}\left(T_{k}\right)$

Proof: For $n=1$ this is the definition of $\tilde{D}$. We proceed by induction, assuming that $\tilde{D}_{t_{3}} \cdots \tilde{D}_{t_{n+1}} f\left(T_{k}\right) \in \mathbb{D}_{0}\left(\mathbb{R}_{+}\right)$for some $n \geq 1$, and

$$
\begin{gathered}
\tilde{D}_{t_{2}} \cdots \tilde{D}_{t_{n+1}} f\left(T_{k}\right)=(-1)^{n} f^{(n)}\left(T_{k}\right) 1_{\left[0, T_{k}\right]}\left(t_{2} \vee \cdots \vee t_{n+1}\right) \\
+(-1)^{n} \sum_{j=1}^{n-1} 1_{\left\{t_{2} \vee \cdots \vee t_{j+1}<t_{j+2} \vee \cdots \vee t_{n+1}\right\}} f^{(n-j)}\left(t_{j+2} \vee \cdots \vee t_{n+1}\right) \delta_{t_{j+2} \vee \cdots \vee t_{n+1}}^{(j-1)}\left(T_{k}\right) .
\end{gathered}
$$

Let for $\varepsilon>0$. We define a smooth approximation of $\tilde{D}_{t_{2}} \cdots \tilde{D}_{t_{n+1}} f\left(T_{k}\right)$ by letting

$$
\begin{array}{r}
F_{\varepsilon}\left(t_{2}, \ldots, t_{n+1}\right)=(-1)^{n} f^{(n)}\left(T_{k}\right) \phi_{\varepsilon} * r^{(1)}\left(T_{k}, t_{2} \vee \cdots \vee t_{n+1}\right) \\
+(-1)^{n} \sum_{j=1}^{n-1} 1_{\left\{t_{2} \vee \cdots \vee t_{j+1}<t_{j+2} \vee \cdots \vee t_{n+1}\right\}} f^{(n-j)}\left(t_{j+2} \vee \cdots \vee t_{n+1}\right) \\
\times \phi_{\varepsilon} * r^{(j+1)}\left(T_{k}, t_{j+2} \vee \cdots \vee t_{n+1}\right) .
\end{array}
$$


Then $F_{\varepsilon}\left(t_{2}, \ldots, t_{n+1}\right) \in \mathcal{S}_{d}(\Omega)$ and

$$
\begin{aligned}
& \tilde{D}_{t_{1}} F_{\varepsilon}\left(t_{2}, \ldots, t_{n+1}\right) \\
& =(-1)^{n+1} 1_{\left[0, T_{k}\right]}\left(t_{1}\right) \phi_{\varepsilon} * r^{(1)}\left(T_{k}, t_{2} \vee \cdots \vee t_{n+1}\right) f^{(n+1)}\left(T_{k}\right) \\
& +(-1)^{n+1} 1_{\left[0, T_{k}\right]}\left(t_{1}\right) f^{(n)}\left(T_{k}\right) \phi_{\varepsilon}^{\prime} * r^{(1)}\left(T_{k}, t_{2} \vee \cdots \vee t_{n+1}\right) \\
& +(-1)^{n+1} 1_{\left[0, T_{k}\right]}\left(t_{1}\right) \sum_{j=1}^{n-1} 1_{\left\{t_{2} \vee \cdots \vee t_{j+1}<t_{j+2} \vee \cdots \vee t_{n+1}\right\}} f^{(n-j)}\left(t_{j+2} \vee \cdots \vee t_{n+1}\right) \\
& \times \phi_{\varepsilon}^{\prime} * r^{(j+1)}\left(T_{k}, t_{j+2} \vee \cdots \vee t_{n+1}\right) \\
& =(-1)^{n+1} 1_{\left[0, T_{k}\right]}\left(t_{1}\right) \phi_{\varepsilon} * r^{(1)}\left(T_{k}, t_{2} \vee \cdots \vee t_{n+1}\right) f^{(n+1)}\left(T_{k}\right) \\
& +(-1)^{n+1} 1_{\left[0, T_{k}\right]}\left(t_{1}\right) f^{(n)}\left(T_{k}\right) \phi_{\varepsilon} * r^{(2)}\left(T_{k}, t_{2} \vee \cdots \vee t_{n+1}\right) \\
& +(-1)^{n+1} 1_{\left[0, T_{k}\right]}\left(t_{1}\right) \sum_{j=2}^{n} 1_{\left\{t_{2} \vee \cdots \vee t_{j}<t_{j+1} \vee \cdots \vee t_{n+1}\right\}} f^{(n+1-j)}\left(t_{j+1} \vee \cdots \vee t_{n+1}\right) \\
& \times \phi_{\varepsilon} * r^{(j+1)}\left(T_{k}, t_{j+1} \vee \cdots \vee t_{n+1}\right) \\
& =(-1)^{n+1} 1_{\left[0, T_{k}\right]}\left(t_{1}\right) \phi_{\varepsilon} * r^{(1)}\left(T_{k}, t_{2} \vee \cdots \vee t_{n+1}\right) f^{(n+1)}\left(T_{k}\right) \\
& +(-1)^{n+1} 1_{\left[0, T_{k}\right]}\left(t_{1}\right) \sum_{j=1}^{n} 1_{\left\{t_{2} \vee \cdots \vee t_{j}<t_{j+1} \vee \cdots \vee t_{n+1}\right\}} f^{(n+1-j)}\left(t_{j+1} \vee \cdots \vee t_{n+1}\right) \\
& \times \phi_{\varepsilon} * r^{(j+1)}\left(T_{k}, t_{j+1} \vee \cdots \vee t_{n+1}\right),
\end{aligned}
$$

where we used the relation $\phi_{\varepsilon}^{\prime} * r^{(j+1)}=\phi_{\varepsilon} * r^{(j+2)}$. As $\varepsilon \rightarrow 0$, $\tilde{D}_{t_{1}} F_{\varepsilon}\left(t_{2}, \ldots, t_{n+1}\right)$ converges in $\mathcal{S}_{d}^{\prime}(\Omega)$ to

$$
\begin{aligned}
& (-1)^{n+1} f^{(n+1)}\left(T_{k}\right) 1_{\left[0, T_{k}\right]}\left(t_{1} \vee \cdots \vee t_{n+1}\right) \\
& +(-1)^{n+1} \sum_{j=1}^{n} 1_{\left\{t_{1} \vee \cdots \vee t_{j}<t_{j+1} \vee \cdots \vee t_{n+1}\right\}} f^{(n+1-j)}\left(t_{j+1} \vee \cdots \vee t_{n+1}\right) \\
& \quad \times r^{(j+1)}\left(T_{k}, t_{j+1} \vee \cdots \vee t_{n+1}\right)
\end{aligned}
$$


In particular, for $n \geq 2$ :

$$
\begin{aligned}
\tilde{D}_{t_{1}} \cdots \tilde{D}_{t_{n}} T_{k} & =(-1)^{n+1} 1_{\left\{0 \leq t_{1}, \ldots, t_{n-1}<t_{n}\right\}} r^{(n)}\left(T_{k}, t_{n}\right) \\
& =(-1)^{n} 1_{\left\{0 \leq t_{1}, \ldots, t_{n-1}<t_{n}\right\}} \delta_{t_{n}}^{(n-2)}\left(T_{k}\right) .
\end{aligned}
$$

We note that since $f \in \mathcal{C}_{b}^{\infty}\left(\mathbb{R}_{+}\right)$, there exists $C>0$ such that

$$
\begin{aligned}
& \left|\left\langle\tilde{D}_{t_{1}} \cdots \tilde{D}_{t_{n}} f\left(T_{k}\right), h_{1} \otimes \cdots \otimes h_{l} \otimes G\right\rangle\right| \\
& \leq C \sum_{i=0}^{i=n}\left\|h_{1} \otimes \cdots \otimes h_{l}\right\|_{\infty}\left\|\hat{D}^{i} G\right\|_{L^{\infty}\left(\Omega, L^{2}\left(\mathbb{R}_{+}, d N_{t}\right) \otimes i\right)},
\end{aligned}
$$

$d t_{1} \cdots d t_{n}$-a.e., for all $G \in \mathcal{S}_{d}(\Omega)$ and $h_{1}, \ldots, h_{l} \in \mathcal{C}_{c}\left(\mathbb{R}_{+}\right)$. Hence $\tilde{D}^{n} f\left(T_{k}\right) \in \mathbb{D}_{n}\left(\mathbb{R}_{+}\right) \subset \mathcal{S}_{d}^{\prime}\left(\Omega \times \mathbb{R}_{+}^{n}\right)$, and (5) can be written as

$$
\begin{aligned}
& \tilde{D}_{t_{1}} \cdots \tilde{D}_{t_{n}} f\left(T_{k}\right) \\
& =(-1)^{n} \sum_{j=0}^{j=n} 1_{\left\{t_{1} \vee \cdots \vee t_{j}<t_{j+1} \vee \cdots \vee t_{n}\right\}} f^{(n-j)}\left(T_{k, j}\right)\left(-r^{(j+1)}\left(T_{k}, t_{j+1} \vee \cdots \vee t_{n}\right)\right)
\end{aligned}
$$

with $t_{0}=0$ and

$$
T_{k, j}= \begin{cases}T_{k} & \text { if } j=0 \\ t_{j+1} \vee \cdots \vee t_{n} & \text { if } j \geq 1\end{cases}
$$

i.e. if $0 \leq t_{1}<\cdots<t_{n}$ :

$$
\tilde{D}_{t_{1}} \cdots \tilde{D}_{t_{n}} f\left(T_{k}\right)=(-1)^{n} \sum_{j=0}^{j=n} f^{(n-j)}\left(T_{k, j}\right)\left(-r^{(j+1)}\left(T_{k}, t_{n}\right)\right) .
$$

If $t_{1}>\cdots>t_{n}$ then

$$
\begin{aligned}
& \tilde{D}_{t_{1}} \cdots \tilde{D}_{t_{n}} F \\
& =(-1)^{n} \sum_{1 \leq j_{1}, \ldots, j_{n} \leq d} 1_{\left[0, T_{j_{1}}\right]}\left(t_{1}\right) \cdots 1_{\left[0, T_{j_{n}}\right]}\left(t_{n}\right) \partial_{j_{1}} \cdots \partial_{j_{n}} f\left(T_{1}, \ldots, T_{d}\right) .
\end{aligned}
$$

Given $j_{1}, \ldots, j_{n} \in\{1, \ldots, d\}$ and $i \in\{1, \ldots, d\}$, let

$$
\begin{aligned}
& a_{i}\left(j_{1}, \ldots, j_{n}\right)=\operatorname{Card}\left\{l \in\{1, \ldots, n\}: j_{l}=i\right\}, \\
& c_{i}\left(j_{1}, \ldots, j_{n}\right)=\max \left\{l: j_{l}=i\right\} .
\end{aligned}
$$

With this notation we obtain the following formula, in which the indices $j_{1}, \ldots, j_{n}$ are omitted in $a_{i}\left(j_{1}, \ldots, j_{n}\right)$ and $c_{i}\left(j_{1}, \ldots, j_{n}\right)$. 
Theorem 1. Let $F=f\left(T_{1}, \ldots, T_{d}\right)$, with $f \in \mathcal{C}_{b}^{\infty}\left(\Delta_{d}\right)$. We have $\tilde{D}^{n} F \in$ $\mathbb{D}_{n}\left(\mathbb{R}_{+}\right)$for all $n \geq 0$, and:

(6) $\tilde{D}_{t_{1}} \cdots \tilde{D}_{t_{n}} F=(-1)^{n} \sum_{\substack{1 \leq j_{1}, \ldots, j_{n} \leq d \\ 0 \leq i_{1} \leq\left(a_{1}-1\right) \vee 0 \\ 0 \leq i_{d} \leq\left(a_{d}-1\right) \vee 0}} \partial_{1}^{a_{1}-i_{1}} \cdots \partial_{d}^{a_{d}-i_{d}} f\left(T_{1, i_{1}}, \ldots, T_{d, i_{d}}\right)$ $\times \prod_{\substack{l=1 \\ a_{l} \neq 0}}^{l=d}\left(-r^{\left(1+i_{l}\right)}\left(T_{l}, t_{c_{l}}\right)\right)$

$\left(t_{1}, \ldots, t_{n}\right) \in \Delta_{n}, n \geq 2$, where

$$
T_{l, i_{l}}= \begin{cases}T_{l} & \text { if } i_{l}=0 \\ t_{c_{l}} & \text { if } i_{l} \geq 1\end{cases}
$$

Proof: Let $\tilde{D}_{t, k}$ denote the partial gradient with respect to the $k$-th variable, i.e.

$$
\tilde{D}_{t, k} f\left(T_{1}, \ldots, T_{d}\right)=-1_{\left[0, T_{k}\right]}(t) \partial_{k} f\left(T_{1}, \ldots, T_{d}\right), \quad 1 \leq k \leq d .
$$

Then $\tilde{D}_{t, k}$ and $\tilde{D}_{s, l}$, are commuting operators, $1 \leq k<l \leq d$, and for $0<t_{1}<\cdots<t_{l}$,

$$
\tilde{D}_{t_{1}, k} \cdots \tilde{D}_{t_{l}, k} F=\left(\tilde{D}_{t_{l}, k}\right)^{l} F \text {. }
$$

Consequently,

$$
\begin{aligned}
\tilde{D}_{t_{1}} \cdots \tilde{D}_{t_{n}} F & =\sum_{1 \leq j_{1}, \ldots, j_{n} \leq d} \tilde{D}_{t_{1}, j_{1}} \cdots \tilde{D}_{t_{n}, j_{n}} F \\
& =\sum_{1 \leq j_{1}, \ldots, j_{n} \leq d}\left(\tilde{D}_{t_{c_{1}}, 1}\right)^{a_{1}} \cdots\left(\tilde{D}_{t_{c_{d}}, d}\right)^{a_{d}} F .
\end{aligned}
$$

It remains to apply Proposition 3 under the form

$$
\begin{aligned}
& \left(\tilde{D}_{t_{c_{l}}, l}\right)^{a_{l}} F \\
= & (-1)^{a_{l}} \sum_{0 \leq i_{l} \leq\left(a_{l}-1\right) \vee 0} \partial_{l}^{a_{l}-i_{l}} f\left(T_{1}, \ldots, T_{l-1}, T_{l, i_{l}}, T_{l+1}, \ldots, T_{d}\right)\left(-r^{\left(i_{l}+1\right)}\left(T_{l}, t_{c_{l}}\right)\right),
\end{aligned}
$$

if $a_{l} \geq 1$, and

$$
\begin{aligned}
& \left(\tilde{D}_{t_{c_{l}}, l}\right)^{a_{l}} \mathrm{~F} \\
& \quad=(-1)^{a_{l}} \sum_{0 \leq i_{l} \leq\left(a_{l}-1\right) \vee 0} \partial_{l}^{a_{l}-i_{l}} f\left(T_{1}, \ldots, T_{l-1}, T_{l, i_{l}}, T_{l+1}, \ldots, T_{d}\right)
\end{aligned}
$$

if $a_{l}=0$. 


\section{Equality of adapted projections in distribution sense}

We recall that the adjoint of $D$ extends the compensated Poisson stochastic integral, cf. [3, Theorem 6.9 , p. 23], i.e. for all adapted squareintegrable process $u \in L^{2}\left(\Omega \times \mathbb{R}_{+}\right)$we have

$$
E\left[\langle D F, u\rangle_{L^{2}\left(\mathbb{R}_{+}, d t\right)}\right]=E\left[F \int_{0}^{\infty} u(t) d\left(N_{t}-t\right)\right] .
$$

Since the adapted projections of $\tilde{D}$ and $D$ coincide, cf. $[\mathbf{7}$, Proposition 20]:

$$
E\left[\tilde{D}_{t} F \mid \mathcal{F}_{a}\right]=E\left[D_{t} F \mid \mathcal{F}_{a}\right], \quad 0<a<t
$$

the same property hold for $\tilde{D}$ :

$$
E\left[\langle\tilde{D} F, u\rangle_{L^{2}\left(\mathbb{R}_{+}, d t\right)}\right]=E\left[F \int_{0}^{\infty} u(t) d\left(N_{t}-t\right)\right] .
$$

We now show that Relation (8) can be extended in distribution sense to $\tilde{D}^{n}$ and $D^{n}, n \geq 2$. The next proposition will be interpreted in terms of generalized conditional expectations as

$E\left[\tilde{D}_{t_{1}} \cdots \tilde{D}_{t_{n}} F \mid \mathcal{F}_{a}\right]=E\left[D_{t_{1}} \cdots D_{t_{n}} F \mid \mathcal{F}_{a}\right],\left(t_{1}, \ldots, t_{n}\right) \in \Delta_{n} \cap\left[a, \infty\left[{ }^{n}\right.\right.$.

Proposition 4. Let $F \in \mathcal{S}_{d}(\Omega)$ and $G \in \mathcal{S}_{d}(\Omega)$ be $\mathcal{F}_{a}$-measurable. We have

(9) $\left\langle G \tilde{D}_{t_{1}} \cdots \tilde{D}_{t_{n}} F, 1\right\rangle=E\left[G D_{t_{1}} \cdots D_{t_{n}} F\right],\left(t_{1}, \ldots, t_{n}\right) \in \Delta_{n} \cap\left[a, \infty\left[{ }^{n}\right.\right.$.

Proof: The proposition holds for $n=1$. We assume that it holds for some $n \geq 1$. Let $F_{\varepsilon}\left(t_{2}, \ldots, t_{n+1}\right)$ denote the regularization of $\tilde{D}_{t_{2}} \cdots \tilde{D}_{t_{n+1}} F$ constructed as in the proof of Proposition 3:

$$
\begin{gathered}
F_{\varepsilon}\left(t_{2}, \ldots, t_{n+1}\right)=(-1)^{n} \sum_{\substack{1 \leq j_{1}, \ldots, j_{n} \leq d \\
0 \leq i_{1} \leq\left(a_{1}-1\right) \vee 0 \\
0 \leq i_{d} \leq\left(a_{d}-1\right) \vee 0}} \partial_{1}^{a_{1}-i_{1}} \cdots \partial_{d}^{a_{d}-i_{d}} f\left(T_{1, i_{1}}, \ldots, T_{d, i_{d}}\right) \\
\qquad \prod_{\substack{l=1 \\
a_{l} \neq 0}}^{l=d}\left(-\phi^{\varepsilon} * r^{\left(1+i_{l}\right)}\left(T_{l}, t_{1+c_{l}}\right)\right) .
\end{gathered}
$$


Let $f_{n+1} \in \mathcal{C}_{c}^{\infty}\left(\Delta_{n+1} \cap\left[a, \infty\left[{ }^{n+1}\right)\right.\right.$. Since $G$ is $\mathcal{F}_{a}$ measurable we have $\tilde{D}_{t_{1}} G=0, t_{1}>a$, hence from Proposition 1 :

$$
\begin{aligned}
E\left[\int_{a}^{t_{2}} \tilde{D}_{t_{1}} F_{\varepsilon}\left(t_{2}, \ldots, t_{n+1}\right) f_{n+1}\left(t_{1}, \ldots, t_{n+1}\right) d t_{1} G\right] \\
=E\left[\int_{a}^{t_{2}} \tilde{D}_{t_{1}}\left(G F_{\varepsilon}\left(t_{2}, \ldots, t_{n+1}\right)\right) f_{n+1}\left(t_{1}, \ldots, t_{n+1}\right) d t_{1}\right] \\
=E\left[G F _ { \varepsilon } ( t _ { 2 } , \ldots , t _ { n + 1 } ) \left(\sum_{k=1}^{k=d} f_{n+1}\left(T_{k}, t_{2}, \ldots, t_{n+1}\right)\right.\right. \\
\left.\left.-\int_{0}^{T_{d}} f_{n+1}\left(t, t_{2}, \ldots, t_{n+1}\right) d t\right)\right]
\end{aligned}
$$

and

$$
\begin{aligned}
& \left\langle\tilde{D}^{n+1} F, 1_{\Delta_{n+1}} f_{n+1} G\right\rangle \\
& =\lim _{\varepsilon \rightarrow 0}\left\langle\int_{a}^{\infty} \int_{a}^{t_{n+1}} \cdots \int_{a}^{t_{2}} \tilde{D}_{t_{1}} F_{\varepsilon}\left(t_{2}, \ldots, t_{n+1}\right) f_{n+1}\left(t_{1}, \ldots, t_{n+1}\right) d t_{1} \cdots d t_{n+1}, G\right\rangle \\
& =\lim _{\varepsilon \rightarrow 0} E\left[\int_{a}^{\infty} \int_{a}^{t_{n+1}} \cdots \int_{a}^{t_{2}} \tilde{D}_{t_{1}} F_{\varepsilon}\left(t_{2}, \ldots, t_{n+1}\right) f_{n+1}\left(t_{1}, \ldots, t_{n+1}\right) d t_{1} \cdots d t_{n+1}, G\right] \\
& =\lim _{\varepsilon \rightarrow 0} E\left[G \int _ { a } ^ { \infty } \int _ { a } ^ { t _ { n + 1 } } \cdots \int _ { a } ^ { t _ { 3 } } F _ { \varepsilon } ( t _ { 2 } , \ldots , t _ { n + 1 } ) \left(\sum_{k=1}^{k=d} f_{n+1}\left(T_{k}, t_{2}, \ldots, t_{n+1}\right)\right.\right. \\
& \left.\left.-\int_{0}^{T_{d}} f_{n+1}\left(t, t_{2}, \ldots, t_{n+1}\right) d t\right) d t_{2} \cdots d t_{n+1}\right] \\
& =E\left[G \int _ { a } ^ { \infty } \int _ { a } ^ { t _ { n + 1 } } \cdots \int _ { a } ^ { t _ { 3 } } \left(\sum_{k=1}^{k=d} f_{n+1}\left(T_{k}, t_{2}, \ldots, t_{n+1}\right)\right.\right. \\
& \left.\left.-\int_{0}^{T_{d}} f_{n+1}\left(t_{1}, t_{2}, \ldots, t_{n+1}\right) d t_{1}\right) \tilde{D}_{t_{2}} \cdots \tilde{D}_{t_{n+1}} F d t_{2} \cdots d t_{n+1}\right] \\
& =E\left[G \int _ { a } ^ { \infty } \int _ { a } ^ { t _ { n + 1 } } \cdots \int _ { a } ^ { t _ { 3 } } \left(\sum_{k=1}^{k=d} f_{n+1}\left(T_{k}, t_{2}, \ldots, t_{n+1}\right)\right.\right. \\
& \left.\left.-\int_{0}^{T_{d}} f_{n+1}\left(t_{1}, t_{2}, \ldots, t_{n+1}\right) d t_{1}\right) D_{t_{2}} \cdots D_{t_{n+1}} F d t_{2} \cdots d t_{n+1}\right] \text {, }
\end{aligned}
$$


where on the last step we used the induction hypothesis with $a=t_{2}$. This is possible because the functional $\sum_{k=1}^{k=d} f_{n+1}\left(T_{k}, t_{2}, \ldots, t_{n+1}\right)-$ $\int_{0}^{T_{d}} f_{n+1}\left(t_{1}, t_{2}, \ldots, t_{n+1}\right) d t_{1}$ is $\mathcal{F}_{t_{2}}$-measurable since it depends on $T_{k}$ only when $T_{k} \leq t_{2}, 1 \leq k \leq d$, due to the fact that $f_{n+1} \in \mathcal{C}_{c}^{\infty}\left(\Delta_{n+1}\right)$.

The proof of Proposition 1 also shows that

$$
\begin{aligned}
E\left[G \left(\sum_{k=1}^{k=d} f_{n+1}\left(T_{k}, t_{2}, \ldots, t_{n+1}\right)\right.\right. & \\
- & \left.\left.\int_{0}^{T_{d}} f_{n+1}\left(t_{1}, t_{2}, \ldots, t_{n+1}\right) d t_{1}\right) D_{t_{2}} \cdots D_{t_{n+1}} F\right] \\
=E & {\left[G \left(\sum_{k=1}^{k=\infty} f_{n+1}\left(T_{k}, t_{2}, \ldots, t_{n+1}\right)\right.\right.} \\
& \left.\left.-\int_{0}^{\infty} f_{n+1}\left(t, t_{2}, \ldots, t_{n+1}\right) d t\right) D_{t_{2}} \cdots D_{t_{n+1}} F\right] \\
= & E\left[G \int_{a}^{t_{2}} f_{n+1}\left(t_{1}, t_{2}, \ldots, t_{n+1}\right) d\left(N_{t_{1}}-t_{1}\right) D_{t_{2}} \cdots D_{t_{n+1}} F\right] \\
=E & {\left[G \int_{a}^{t_{2}} f_{n+1}\left(t_{1}, \ldots, t_{n+1}\right) D_{t_{1}} \cdots D_{t_{n+1}} F d t_{1}\right], }
\end{aligned}
$$

where on the last line we used the duality (7) between $D$ and the Poisson compensated integral on the adapted processes. Hence

$$
\begin{aligned}
& \left\langle\tilde{D}^{n+1} F, 1_{\Delta_{n+1}} f_{n+1} G\right\rangle \\
= & E\left[G \int_{a}^{\infty} \int_{a}^{t_{n+1}} \cdots \int_{a}^{t_{2}} f_{n+1}\left(t_{1}, \ldots, t_{n+1}\right) D_{t_{1}} \cdots D_{t_{n+1}} F d t_{1} \cdots d t_{n+1}\right] .
\end{aligned}
$$

This shows the almost-sure equality

$$
\begin{aligned}
& \left\langle G \tilde{D}_{t_{1}} \cdots \tilde{D}_{t_{n}} F, 1\right\rangle=E\left[G D_{t_{1}} \cdots D_{t_{n}} F\right] \\
& \text { a.e. }\left(t_{1}, \ldots, t_{n}\right) \in \Delta_{n} \cap\left[a, \infty\left[^{n},\right.\right.
\end{aligned}
$$


which becomes an equality for all $\left(t_{1}, \ldots, t_{n}\right) \in \Delta_{n} \cap\left[a, \infty\left[{ }^{n}\right.\right.$ since $\left(t_{1}, \ldots, t_{n}\right) \mapsto\left\langle G \tilde{D}_{t_{1}} \cdots \tilde{D}_{t_{n}} F, 1\right\rangle$ and $\left(t_{1}, \ldots, t_{n}\right) \mapsto E\left[G D_{t_{1}} \cdots D_{t_{n}} F\right]$ are clearly continuous functions on $\Delta_{n}$ when $F, G \in \mathcal{S}_{d}(\Omega)$.

Note that Relation (9) does not hold if $\left(t_{1}, \ldots, t_{n}\right) \notin \Delta_{n}$, see Relation (14) below.

\section{Chaos expansions of jump times functionals}

Our result is stated for smooth functions $f\left(T_{1}, \ldots, T_{d}\right)$ of a finite number of jump times. We start with the simple case of $f\left(T_{d}\right)$. For $n \in \mathbb{Z}$ and $t \in \mathbb{R}_{+}$, let

$$
p_{n}(t)=P\left(N_{t}=n\right)=\frac{t^{n}}{n !} e^{-t}=e^{-t} \int_{0}^{t} \int_{0}^{s_{n-1}} \cdots \int_{0}^{s_{2}} d s_{1} \cdots d s_{n-1}
$$

if $n \geq 0$, and $p_{n}(t)=P\left(N_{t}=n\right)=0$ if $n<0$, i.e. $p_{n-1}: \mathbb{R}_{+} \rightarrow \mathbb{R}_{+}$, $n \geq 1$, is the density function of $T_{n}$, and

$$
p_{n}^{(k)}(t)=\frac{\partial^{k}}{\partial t^{k}} p_{n}(t)=(-\Delta)^{k} p_{n}(t)=(-1)^{k} \frac{p_{n}(t)}{t^{k}} C_{k}^{t}(n),
$$

where $\Delta$ is the finite difference operator $\Delta f(n)=f(n)-f(n-1)$ and $C_{k}^{t}$ is the Charlier polynomial of order $k \in \mathbb{N}$ and parameter $t \in \mathbb{R}_{+}$.

Proposition 5. The decomposition

$$
f\left(T_{d}\right)=\sum_{n=0}^{\infty} I_{n}\left(h_{n} 1_{\Delta_{n}}\right)
$$

is given for $\left(t_{1}, \ldots, t_{n}\right) \in \Delta_{n}$ as:

$$
\begin{aligned}
& h_{n}\left(t_{1}, \ldots, t_{n}\right) \\
= & (-1)^{n} \int_{t_{n}}^{\infty} f^{(n)}(t) p_{d-1}(t) d t+(-1)^{n} \sum_{j=1}^{j=n-1}(-1)^{j-1} f^{(n-j)}\left(t_{n}\right) p_{d-1}^{(j-1)}\left(t_{n}\right) .
\end{aligned}
$$


Proof: From Relation (9) of Proposition 4 and Relation (5) of Proposition 3 we have

$$
\begin{aligned}
& h_{n}\left(t_{1}, \ldots, t_{n}\right)=E\left[D_{t_{1}} \cdots D_{t_{n}} f\left(T_{d}\right)\right]=\left\langle\tilde{D}_{t_{1}} \cdots \tilde{D}_{t_{n}} f\left(T_{d}\right), 1\right\rangle \\
& =(-1)^{n}\left\langle f^{(n)}\left(T_{d}\right) 1_{\left[0, T_{d}\right]}\left(t_{n}\right)+\sum_{j=1}^{n-1} f^{(n-j)}\left(t_{n}\right) \delta_{t_{n}}^{(j-1)}\left(T_{d}\right), 1\right\rangle \\
& =(-1)^{n} \int_{0}^{\infty} 1_{\left[t_{n}, \infty[\right.}\left(s_{d}\right) e^{-s_{d}} f^{(n)}\left(s_{d}\right) \int_{0}^{s_{d}} \cdots \int_{0}^{s_{2}} d s_{1} \cdots d s_{d} \\
& \quad+(-1)^{n} \sum_{j=1}^{n-1} f^{(n-j)}\left(t_{n}\right) \int_{0}^{\infty} e^{-s_{d}} \int_{0}^{s_{d}} \cdots \int_{0}^{s_{2}} d s_{1} \cdots d s_{d-1} \delta_{t_{n}}^{(j-1)}\left(d s_{d}\right) \\
& =(-1)^{n} \int_{t_{n}}^{\infty} e^{-s_{d}} f^{(n)}\left(s_{d}\right) \frac{s_{d}^{d-1}}{(d-1) !} d s_{d}{ }^{n-1} f^{(n-j)}\left(t_{n}\right) \int_{0}^{\infty} e^{-s_{d}} \frac{s_{d}^{d-1}}{(d-1) !} \delta_{t_{n}}^{(j-1)}\left(d s_{d}\right) \\
& \quad+(-1)^{n} \sum_{j=1}^{\infty-1} \int_{t_{n}}^{\infty} f^{(n)}(t) p_{d-1}(t) d t \\
& \quad+(-1)^{n} \sum_{j=1}^{j=n-1}(-1)^{j-1} f^{(n-j)}\left(t_{n}\right) p_{d-1}^{(j-1)}\left(t_{n}\right) .
\end{aligned}
$$

By induction this gives for $l=0, \ldots, n-1$ :

$$
\begin{aligned}
h_{n}\left(t_{1}, \ldots, t_{n}\right)=(-1)^{n+l} & \int_{t_{n}}^{\infty} f^{(n-l)}(t) p_{d-1}^{(l)}(t) d t \\
& +(-1)^{n} \sum_{j=l+1}^{j=n-1}(-1)^{j-1} f^{(n-j)}\left(t_{n}\right) p_{d-1}^{(j-1)}\left(t_{n}\right),
\end{aligned}
$$

and in particular for $l=n-1$ :

$$
\begin{aligned}
h_{n}\left(t_{1}, \ldots, t_{n}\right) & =-\int_{t_{n}}^{\infty} f^{\prime}(s) p_{d-1}^{(n-1)}(s) d s \\
& =f\left(t_{n}\right) p_{d-1}^{(n-1)}\left(t_{n}\right)+\int_{t_{n}}^{\infty} f(s) p_{d-1}^{(n)}(s) d s
\end{aligned}
$$


hence

$$
\begin{array}{r}
f\left(T_{d}\right)=\sum_{n \geq 0} \frac{1}{n !} I_{n}\left(f\left(t_{1} \vee \cdots \vee t_{n}\right) p_{d-1}^{(n-1)}\left(t_{1} \vee \cdots \vee t_{n}\right)\right. \\
\left.+\int_{t_{1} \vee \cdots \vee t_{n}}^{\infty} f(s) p_{d-1}^{(n)}(s) d s\right)
\end{array}
$$

with the convention $t_{1} \vee t_{0}=0$. In order to treat the case of $d$ variables we recall the notation

$$
a_{i}\left(j_{1}, \ldots, j_{n}\right)=\operatorname{Card}\left\{l: j_{l}=i\right\} \text {, and } c_{i}\left(j_{1}, \ldots, j_{n}\right)=\max \left\{l: j_{l}=i\right\} \text {. }
$$

Proposition 6. Let $f \in \mathcal{C}_{b}^{\infty}\left(\Delta_{d}\right)$. We have

$$
f\left(T_{1}, \ldots, T_{d}\right)=\sum_{n=0}^{\infty} I_{n}\left(h_{n} 1_{\Delta_{n}}\right),
$$

with

(11) $h\left(t_{1}, \ldots, t_{n}\right)$

$$
\begin{gathered}
=(-1)^{n} \sum_{\substack{1 \leq j_{1}, \ldots, j_{n} \leq d \\
0 \leq i_{1} \leq\left(a_{1}-1\right) \vee 0 \\
0 \leq i_{d} \leq\left(a_{d}-1\right) \vee 0}} \int_{0}^{\infty} \int_{0}^{s_{d}} \cdots \int_{0}^{s_{2}} e^{-s_{d}} \partial_{1}^{a_{1}-i_{1}} \cdots \partial_{d}^{a_{d}-i_{d}} f\left(s_{1, i_{1}}, \ldots, s_{d, i_{d}}\right) \\
\times \prod_{\substack{l=1 \\
a_{l} \neq 0}}^{l=d}\left(-r^{\left(1+i_{l}\right)}\left(d s_{l}, t_{c_{l}}\right)\right),
\end{gathered}
$$

where $r^{(1)}(d s, t)=1_{[t, \infty[}(s) d s$, and

$$
s_{l, i_{l}}= \begin{cases}s_{l} & \text { if } i_{l}=0, \\ t_{c_{l}} & \text { if } i_{l} \geq 1, l=1, \ldots, d .\end{cases}
$$

Proof: We apply Theorem 1 and Relation (9) of Proposition 4.

This expression can be made more explicit by evaluation of the action of $r^{\left(1+i_{l}\right)}\left(d s_{j_{l}}, t_{c_{l}}\right)$, either as an indicator function or as a derivative in $t_{c_{l}}$. However this will not be done here in order to keep formula (11) to a reasonable size.

In [8], another expression (different from (11)) has been obtained using elementary orthogonal decompositions in Charlier polynomials. Let $n_{1}, \ldots, n_{l} \in \mathbb{N}$ with $1 \leq n_{1}<\cdots<n_{l}$, and let $f \in \mathcal{C}_{b}^{d}\left(\Delta_{l}\right)$. As a 
convention, if $k_{1} \geq 0, \ldots, k_{d} \geq 0$ satisfy $k_{1}+\cdots+k_{d}=n$, we let for $\left(t_{1}, \ldots, t_{n}\right) \in \Delta_{n}$ :

$$
\left(t_{1}^{1}, \ldots, t_{k_{1}}^{1}, t_{1}^{2}, \ldots, t_{k_{2}}^{2}, \ldots, t_{1}^{d}, \ldots, t_{k_{d}}^{d}\right)=\left(t_{1}, \ldots, t_{n}\right),
$$

with $t_{k_{i}}^{i}=0$ if $k_{i}=0$, and $\left(t_{1}^{i}, t_{0}^{i}\right)=()$. We have

$$
f\left(T_{n_{1}}, \ldots, T_{n_{l}}\right)=\sum_{n=0}^{\infty} I_{n}\left(1_{\Delta_{n}} h_{n}\right)
$$

where

$$
\begin{aligned}
& \text { (12) } h_{n}\left(t_{1}, \ldots, t_{n}\right) \\
& =(-1)^{l} \sum_{k_{1}+\cdots+k_{l}=n} \int_{t_{k_{l}}^{l}}^{\infty} \cdots \int_{t_{k_{i}}^{i}}^{t_{1}^{i+1}} \ldots \int_{t_{k_{1}}^{1}}^{t_{1}^{2}} \partial_{1} \cdots \partial_{l} f\left(s_{1}, \ldots, s_{l}\right) K_{s_{1}, \ldots, s_{l}}^{k_{1}, \ldots, k_{l}} \\
& d s_{1} \cdots d s_{l},
\end{aligned}
$$

with, for $0 \leq s_{1} \leq \cdots \leq s_{l}$ and $k_{1} \geq 0, \ldots, k_{l} \geq 0$ :

$$
\begin{array}{r}
K_{s_{1}, \ldots, s_{l}}^{k_{1}, \ldots, k_{l}}=\sum_{\substack{m_{1} \geq n_{1}, \ldots, m_{l} \geq n_{l} \\
m_{1} \leq \ldots \leq m_{l}}} p_{m_{1}-m_{0}}^{\left(k_{1}\right)}\left(s_{1}-s_{0}\right) \cdots p_{m_{l}-m_{l-1}}^{\left(k_{l}\right)}\left(s_{l}-s_{l-1}\right), \\
m_{0}=0, \quad s_{0}=0,
\end{array}
$$

cf. Theorem 1 of $[8]$. For $l=1$, i.e. for $f\left(T_{d}\right), n_{1}=d$, we have

$$
\begin{aligned}
K_{s}^{k} & =\sum_{m=d}^{\infty} p_{m}^{(k)}(s)=\frac{\partial^{k-1}}{\partial s^{k-1}} \sum_{m=d}^{\infty} p_{m}^{(1)}(s) \\
& =\frac{\partial^{k-1}}{\partial s^{k-1}} \sum_{m=d}^{\infty} p_{m-1}(s)-p_{m}(s)=p_{d-1}^{(k-1)}(s)
\end{aligned}
$$

hence

$$
h_{n}\left(t_{1}, \ldots, t_{n}\right)=-\int_{t_{n}}^{\infty} f^{\prime}(s) p_{d-1}^{(n-1)}(s) d s,
$$

which coincides with (10).

Remarks. i) All expressions obtained above for $f\left(T_{1}, \ldots, T_{d}\right), f \in$ $\mathcal{C}_{b}^{\infty}\left(\Delta_{d}\right)$, extend to $f \in L^{2}\left(\Delta_{d}, e^{-s_{d}} d s_{1} \cdots d s_{d}\right)$, i.e. to squareintegrable $f\left(T_{1}, \ldots, T_{d}\right)$, by repeated integrations by parts.

ii) Chaotic decompositions on the Poisson space on the compact interval $[0,1]$ as in $[\mathbf{4}]$ or $[\mathbf{5}]$ can be obtained by considering the functional $f\left(1 \wedge T_{1}, \ldots, 1 \wedge T_{d}\right)$ instead of $f\left(T_{1}, \ldots, T_{d}\right)$. 
iii) If $t_{1}>\cdots>t_{n}$ then Relation (9) does not hold, for example we have

$$
\tilde{D}_{t_{1}} \cdots \tilde{D}_{t_{n}} f\left(T_{d}\right)=(-1)^{n} 1_{\left[0, T_{d}\right]}\left(t_{1}\right) f^{(n)}\left(T_{d}\right)
$$

and

$$
\begin{aligned}
E\left[\tilde{D}_{t_{1}} \cdots \tilde{D}_{t_{n}} f\left(T_{d}\right)\right] & =(-1)^{n} E\left[1_{\left[0, T_{d}\right]}\left(t_{1}\right) f^{(n)}\left(T_{d}\right)\right] \\
& =(-1)^{n} \int_{t_{1}}^{\infty} f^{(n)}(s) p_{d-1}(s) d s,
\end{aligned}
$$

which differs (if $n \geq 2$ ) from

$$
E\left[D_{t_{1}} \cdots D_{t_{n}} f\left(T_{d}\right)\right]=-\int_{t_{1}}^{\infty} f^{\prime}(s) p_{d-1}^{(n-1)}(s) d s .
$$

\section{References}

[1] E. A. Carlen and E. Pardoux, Differential calculus and integration by parts on Poisson space, in: "Stochastics, algebra and analysis in classical and quantum dynamics" (Marseille, 1988), Math. Appl. 59, Kluwer Acad Publ., Dordrecht, 1990, pp. 63-73.

[2] R. J. Elliott and A. H. Tsoi, Integration by parts for Poisson processes, J. Multivariate Anal. 44(2) (1993), 179-190.

[3] Y. Iто, Generalized Poisson functionals, Probab. Theory Related Fields 77(1) (1988), 1-28.

[4] J. A. León, J. L. Solé and J. Vives, The chaos decomposition of functionals of the jump times for the Poisson process over a compact time interval, in: "Stochastic models" (Guanajuato, 1998), Aportaciones Mat. Investig. 14, Soc. Mat. Mexicana, México, 1998, pp. 269-282.

[5] J. A. León And C. Tudor, The chaos decomposition of the jump times for the Poisson process over a compact time interval, in: "Fourth Symposium on Probability Theory and Stochastic Processes" (Guanajuato, 1996), Aportaciones Mat. Notas Investigación 12, Soc. Mat. Mexicana, México, 1996, pp. 137-146.

[6] D. Nualart and J. Vives, Anticipative calculus for the Poisson process based on the Fock space, in: "Séminaire de Probabilités, XXIV, 1988/89", Lecture Notes in Math. 1426, Springer, Berlin, 1990, pp. 154-165.

[7] N. Privault, Chaotic and variational calculus in discrete and continuous time for the Poisson process, Stochastics Stochastics Rep. 51(1-2) (1994), 83-109. 
[8] N. Privault, Multiple stochastic integral expansions of arbitrary Poisson jump times functionals, Statist. Probab. Lett. 43(2) (1999), 179-188.

[9] N. Privault, Connection, parallel transport, curvature and energy identities on spaces of configurations, C. R. Acad. Sci. Paris Sér. I Math. 330(10) (2000), 899-904.

Département de Mathématiques

Université de La Rochelle

Avenue Michel Crépeau

17042 La Rochelle Cedex 1

France

E-mail address: nprivaul@univ-lr.fr

Primera versió rebuda el 6 de febrer de 2001, darrera versió rebuda el 15 de setembre de 2001. 